\title{
La psicogénesis del delirio en la obra y en la época de E. Kretschmer
}

RESUMEN: Se estudian los principales aspectos de la obra de Kretschmer El delirio sensitivo de referencia y se comparan con los de autores de la misma época que abordan la psicogénesis del delirio, como Jaspers, Gaupp, Bleuler, Sérieux y Capgras y Freud.

PALABRAS CLAVE: Paranoia, delirio, psicogénesis, personalidad.
SUMMARY: The main aspects of The Sensitive Delusion of Reference of by Kretschmer are studied and compared with authors of the same period such as Jaspers, Gaupp, Bleuler, Sérieux and Capgras and Freud who discuss the psychogenesis of delusion.

KEY WORDS: Paranoia, Delusion, Psychogenesis, Personality.

\section{Introducción}

Hace algunos meses se reeditó en castellano El delirio sensitivo de referencia, de Kretschmer (1). Se trata de un libro que puede interesar a muchos, sobre todo a aquellos que, dedicados fundamentalmente a la clínica, echamos de menos en la psiquiatría actual textos que contengan tantas enseñanzas como en este aspecto contiene éste. Aparte de su cuantiosa y exhaustiva casuística, que hace su lectura especialmente recomendable para aquellos que se inician en su actividad profesional como psiquiatras o psicólogos clínicos, el libro es importante por otras muchas razones. Se puede considerar mérito de Kretschmer, aunque no solo de él, haber roto con el corsé que la nosología kraepeliniana trató de imponer en la psiquiatría clínica de principios del siglo XX. Como se sabe, Kraepelin pretendió construir una nosología psiquiátrica mediante la delimitación de "unidades" clínicas supuestamente naturales; así, en la $8^{\text {a }}$ edición de su tratado, de 1899, distingue tres enfermedades "endógenas": la demencia precoz, la locura maníaco-depresiva y la paranoia. Por tanto, inicialmente, la paranoia fue considerada por Kraepelin de "causa interna", es decir, no psicógena; separada de ella situó al delirio querulante, al que sí consideró reactivo a una situación externa. Pues bien, la obra de Kretschmer rompe estas rígidas fronteras entre endogeneidad y psicogénesis, e insiste en la comprensibilidad del delirio paranoico y en la posibilidad de una evolución favorable del mismo.

Como con anterioridad hiciera su maestro Gaupp en la descripción del caso Wagner (vid. 5.1) (2), Kretschmer pretendió hacer ver que el delirio constituye una defensa frente a determinados conflictos internos que son movilizados en el transcurso de las interacciones del sujeto con su medio social. Se trata, por tanto, de un síntoma perfectamente "comprensible" si se tiene en cuenta su contexto natural, la personalidad, el modo de ser, determinado a su vez por la historia de las relaciones del sujeto con Rev. Asoc. Esp. Neuropsiq., 2001, vol XXI, n. ${ }^{\circ}$ 80, pp. 73-97 
la realidad, es decir, por su biografía. En esto, precisamente, radica el principal cometido del libro: mostrar las relaciones entre la estructura de la personalidad, el delirio y ciertos acontecimientos vitales conflictivos para el sujeto, así como la delimitación de determinadas psicosis accesibles a la psicoterapia (3). En virtud de esto, se puede decir que la posición epistemológica que adopta Kretschmer es encuadrable en la hermenéutica, en el sentido que le diera Dilthey.

La hermenéutica o tradición de la Verstehen (comprensión) constituyó una reacción frente al monismo metodológico positivista del siglo XIX que, en psiquiatría, quedó plasmado en el axioma de Griessinger "Las enfermedades mentales son enfermedades del cerebro". Influyó en el pensamiento psiquiátrico de la primera mitad del siglo XX a través de la psicopatología del Jaspers, introductor del concepto de comprensión de Dilthey en psicopatología. Las figuras más destacadas de esta tendencia son Droysen, Dilthey, Simmel y Max Weber; asimismo, cercanos a ellos son Windelband y Rickert; por ejemplo, Windelband propuso los términos nomotético para calificar las ciencias que buscan el establecimiento de leyes, e idiográfico para aludir a la descripción de lo individual. En un sentido similar, los términos explicación (Erklären) y comprensión (Verstehen), que aluden a los métodos de las ciencias naturales y humanas respectivamente, son de Droysen, pero es en Dilthey donde alcanzan su mayor sistematización (4). El psicoanálisis, aunque desde una axiomática particular, pertenece también a esta tradición, pues como dice López Ibor en el prólogo del libro de Martín Santos Dilthey, Jaspers y la comprensión del enfermo mental, “QQué es la psicodinámica, tan en boga actualmente, sino un fabuloso intento de ensanchar los límites de la comprensibilidad de la vida psíquica anormal?" (5).

La primera edición en alemán de El delirio sensitivo de referencia es de 1918. Inicialmente constaba de 10 capítulos; en 1949, el autor le añadió uno más: "Estructuras instintivas y predisposición endógena". La primera edición española, traducción de la $3^{\mathrm{a}}$ alemana, es de 1949. La actual edición en Triacastela es traducción de la $4^{\text {a }}$ edición alemana, de 1966, al cuidado de su hijo Wolfgang Kretschmer, quien realiza una breve introducción y añade un interesante capítulo sobre "Concepto, historia y situación científica del delirio sensitivo de referencia".

En el presente trabajo se exponen las líneas fundamentales del libro y se comparan con las de algunas de las principales obras sobre el delirio paranoico publicadas en los primeros años del siglo XX.

\section{Psicogénesis y comprensión.}

No entraremos aquí, pues no es el tema del presente trabajo, en la inveterada discusión acerca de las relaciones entre lo físico y lo mental, entre el cerebro y la mente, que llevada al campo de la patogenia se traduce en los conceptos de somatogénesis y psicogénesis. En lo que respecta a la etiología de las enfermedades mentales, la discusión es ya antigua (6); por ejemplo, la sostenida hasta mediados del siglo XIX entre los denominados 
HISTORIA

Psychiker y Somatiker, esto es, entre quienes consideraban la locura como una enfermedad del alma, sea como desviación de los principios divinos, sea de los éticos, y los que, partiendo de la base de que el alma es inalienable y que, por lo tanto, la mente solo es susceptible de ser alterada por una causa corporal, la entendían como síntoma de un disturbio orgánico (7) (8). En lo que se refiere a esta cuestión, en 1918, fecha en que se publica El delirio sensitivo (a partir de ahora DS), estaban en uso las teorías constitucionalistas, de tal modo que, como se sabe, el mismo Kretschmer, en una obra posterior a la que aquí estamos comentando, Constitución y carácter (9), trató de establecer correlaciones supuestamente generadas por vías endocrinohumorales y neurovegetativas, entre hábitos físicos y formas de ser; así, el carácter ciclotímico se correspondía con el biotipo pícnico y el esquizotímico con el leptosómico.

Las doctrinas constitucionalistas postulan la existencia de factores individuales innatos que determinan la estructura de la personalidad. Como más adelante veremos ( $c f r .5 .3)$, algunos autores de la época que estamos tratando vieron en una supuesta constitución paranoica la predisposición a sufrir determinados tipos de delirio; pero, como dice Lacan, "la existencia de una correlación de la psicosis con determinada predisposición constitucional no demuestra por sí misma una determinación psicógena. La constitución, en efecto, puede no traducir sino una fragilidad orgánica con respecto a una causa patógena exterior a la personalidad, es decir, con respecto a cierto proceso psíquico, para emplear el concepto general elaborado por Jaspers, ..." (10) (vid. 2.1)).

Quizá sea oportuno, por lo que afecta al objeto del presente trabajo, hacer una breve digresión acerca de estos conceptos, hoy en día casi en desuso. Por constitución entiende Kretschmer “...el conjunto de todas las cualidades individuales basadas en la herencia, esto es de raigambre genotípica." (11); pero añade este autor que "...nunca podrá separar(se) rigurosamente del concepto de constitución las modificaciones de la predisposición hereditaria ocasionada por estímulos externos (...); pues todo cuanto vive concretamente se nos da como producto de la relación entre la predisposición y el ambiente." (12). Predisposición y constitución son, pues, conceptos equivalentes y, para el autor, eventualmente, abarca también las variaciones de la predisposición, "aún muy plástica en las primeras fases de la vida", provocadas por los estímulos procedentes del ambiente. Lo constitucional es punto de confluencia de lo somático y lo psíquico, es un concepto psicofísico; esto lo diferencia del concepto de carácter, que es puramente psicológico: "Entendemos por carácter la totalidad de las posibilidades afectivovolitivas de reacción de una persona, surgidas en el curso de su evolución vital, o sea, a partir de la predisposición hereditaria y de todos los factores exógenos: influjos físicos, educación, ambiente y huellas incidentales o episódicas" (13). Jaspers distingue la constitución física: "el todo de la vida corporal", lo que vincula entre sí a las funciones corporales, esto es, el hábito, y la constitución psíquica: 
cantidad de energía psíquica, excitabilidad, fatigabilidad, modo de reacción, etc. (14).

Ahora bien, ¿qué se entiende por psicogénesis? Para Lacan, autor que en su tesis doctoral, publicada en 1932, estudió exhaustivamente la psicogénesis del delirio, "Es psicógeno un síntoma -físico o mental- cuyas causas se expresan en función de los mecanismos complejos de la personalidad, cuya manifestación los refleja y cuyo tratamiento puede depender de ellos." (15). Esto es: 1) está determinado por la biografía del sujeto, por la concepción que de sí mismo tenga y por su situación vital; 2) es reflejo de la biografía del sujeto o expresa contenidos de sus deseos, y 3) el tratamiento depende de la modificación de la situación del sujeto, ya sea por la de los hechos, sea por la relación afectiva con ellos o ya sea por la representación que se hace de ellos. Queda así delimitado el campo de lo psicógeno a los trastornos derivados de lo que este autor denomina síntesis psíquica, esto es, la personalidad, que "se nos muestra como el elemento de síntesis de nuestra experiencia interior." (16). Para nosotros, resumidamente dicho, lo psíquico se refiere a la actividad del sujeto, a la conducta en cuanto actividad con sentido, es decir, motivación y/o intencionalidad. Esta concepción de la conducta tiene sus orígenes en la filosofía hermenéutica, como ya hemos visto, $\mathrm{y}$, en psiquiatría, en la psicología comprensiva de Jaspers (17). Una visión hermenéutica, interpretativa, de la conducta implica teorías acerca de sus motivos, y toda teoría de motivos, como dice Castilla del Pino, es, en última instancia, una teoría acerca del sujeto y de su actuación (18).

Existen varias acepciones del término comprensión; puede ser entendido, en un sentido intuitivo, como empatía, esto es, la capacidad que tiene el sujeto de ponerse en lugar de otro; o también puede entenderse en relación con la intencionalidad de la conducta. Desde esta perspectiva, "Se comprenden los objetivos y propósitos de un agente, el significado de un signo o de un símbolo, el sentido de una institución social o de un acto religioso." (19). Martín Santos (20) distingue varios tipos de comprensión: 1) Comprensión racional, que es de índole lógica, no psicológica, de tal modo que el sentido de lo captado tiene una fundamentación empírica. Así, en el ejemplo de Martín Santos, "Si un individuo dice: 'Dos y dos son cuatro', expresa aquí un sentido racional que yo capto y comprendo" (21). La comprensión racional fue usada en psicopatología por Kurt Schneider, distinguiendo la comprensión del contenido de la comprensión de la forma de la vivencia; de este modo, aplicada a los delirios, una cosa sería el comprender racionalmente el contenido del delirio, otra la forma en que el delirio es vivido y otra el modo cómo se ha producido en la mente del enfermo. 2) Comprensión analógico-simbólica, que Jaspers denominó comprensión como si, dirigida a captar el sentido simbólico que subyace a la vida consciente (22); hay tantos tipos, dice Martín Santos, como teorías sobre el hombre al respecto. Se inicia su aplicación a la psicopatología con el psicoanálisis freudiano, prosigue con Bleuler y Kretschmer, y se continúa, como fase antropológica, en la analítica 
HISTORIA

existencial (Weizsäcker, Biswanger, Strausss, von Gebsattel, etc.). 3) Comprensión psicológica. Se trata de la comprensión de la vida consciente o "comprensión en sentido estricto" que, a su vez, puede ser estática ofenomenológica si se refiere al presente, y dinámica o genética si se refiere a relaciones entre momentos diferentes. La comprensión estática pretende saber de la vida psíquica en un momento dado; en Dilthey, consiste en conectar la vivencia con el resto de la vida psíquica; en esto radica su significado. La comprensión dinámica pretende el establecimiento de relaciones comprensibles, de sentido, entre vivencias separadas en el tiempo en un mismo sujeto. En realidad, ambos tipos de comprensión psicológica no pueden darse aisladas una de la otra, pues la comprensión de la vivencia individual exige la de su significado, esto es, la relación de sentido que conecta cada vivencia (23) (24).

\subsection{Comprensión y delirio en Jaspers.} Jaspers distingue dos tipos de alteraciones psicopatológicas: 1) Alteraciones cuyo desarrollo es comprensible; en ellas enmarca, por un lado, las "reacciones legítimas cuyo contenido está en relación comprensible con la vivencia, que no se habrían producido $\sin$ la vivencia y que dependen en su curso de la vivencia y de sus relaciones." (25); por otro lado está el desarrollo de la personalidad, originado en función de las disposiciones individuales y que evolucionan a través de la vida sin discontinuidad incomprensible. La disposición individual determina la manera de reaccionar frente a los acontecimientos. Este segundo grupo no es totalmente separable del anterior, pues la patología "comprensible" se construye a expensas de cierto grado de predisposición y de cierto grado de reacción. 2) Alteraciones cuyo desarrollo no es comprensible, pues rompen con mayor o menor brusquedad el desarrollo comprensible de la vida psíquica, introduciendo en ella un cambio totalmente nuevo. Esto puede ocurrir por procesos orgánicos de muy diversa índole (cuadros cerebrales tóxicos, infecciosos, etc.); también por procesos psíquicos, en los que se evidencia una alteración marcada de la personalidad, como ocurre en la esquizofrenia; por último, por fases o alteraciones de la vida psíquica, sean puramente endógenas, sean determinadas por acontecimientos accidentales, evolucionando durante un tiempo más o menos largo y desapareciendo de manera que el estado anterior queda restablecido. (26) (27).

Sostiene Berrios (28) que todos los aspectos del delirio atribuidos a Jaspers habían sido ya tratados durante el siglo XIX; no obstante, dada la importancia que poseen las concepciones de Jaspers en lo que respecta al presente trabajo, haremos un resumen de lo fundamental de ellas. Jaspers se refiere al delirio en un apartado de su Psicopatología general titulado "La conciencia de realidad y las ideas delirantes". (29). Citaremos algunos pasajes: "El delirio es un fenómeno primario. Tener esto presente es la tarea primera. El vivenciar dentro del cual tiene lugar el delirio, es la experiencia y el pensamiento de la realidad". (30). Diferencia entre lo real pensado y lo real vivenciado, entre juicio de realidad y vivencia de realidad; el juicio de realidad surge de la elaboración 
de las experiencias directas, mientras que de la vivencia de realidad se obtiene una certeza directa e inmediata: "La vivencia de la realidad no se puede derivar, ni poner en el mismo grado con otros fenómenos afines, sino describir sólo indirectamente como un fenómeno originario." (31). La descripción de este fenómeno ha de tener en cuenta que: 1) la realidad es percibida "corporalmente", esto es, sensorialmente; su cualidad, el "modo de lo sentido" es originario, no derivable. 2) La realidad está en la "conciencia del ser" (lo que Janet denominó fonction du réel); por ello, la conciencia de que existo me permite considerar las cosas de fuera de mí como reales. 3) La realidad nos ofrece "resistencia": se alcanzan objetivos contra ella, o se choca contra esa resistencia. Pero, además, en la práctica, la realidad es la significación de las cosas. Esta significación, para Jaspers, está indisolublemente unidad a la percepción de la realidad, pues el sujeto, en virtud de la cultura a que pertenece, su educación, etc. "cuenta" con ella; esto es, las cosas le son dadas con un significado. De esta "conciencia o vivencia de la realidad" hay que diferenciar el juicio que, secundariamente, mediante una elaboración, el sujeto realiza sobre ella.

Según lo anterior, “(...) el delirio es una transformación en la vasta conciencia de la realidad (que se enuncia secundariamente en juicios de realidad), que se construye sobre esas experiencias, en el mundo de la práctica, de las resistencias y de las significaciones, (...)". (32). El delirio se comunica en juicios pues "Solo donde se piensa y se juzga puede surgir un delirio. En este sentido se llaman ideas delirantes a los juicios patológicamente falseados”. (33). Según su origen, existen dos grandes clases: a las que surgen "comprensiblemente" de fenómenos afectivos, de percepciones falsas, etc., las denomina Jaspers ideas deliroides, mientras que las ideas delirantes que "no pueden ser seguidas psicológicamente más atrás", esto es, no son comprensibles, son "auténticas ideas delirantes" (34). Estas "ideas delirantes primarias" surgen pues de "vivencias delirantes primarias". Se trata de vivencias, dice Jaspers, que no son fáciles de describir; las denomina "temple delirante" (Wahnstimmung), que consiste en una significación alterada de la realidad; si en la vivencia normal la realidad aparece, como antes hemos dicho, ya significada, la vivencia delirante consiste en una alteración de esta significación: "La conciencia de la significación experimenta una transformación radical. El saber inmediato que se impone de las significaciones, es la vivencia primaria del delirio" (35). Para Jaspers, no se trata de una mera interpretación que el sujeto hace de la realidad percibida, "no se trata aquí de interpretaciones conformes al juicio, sino que es experimentada directamente la significación en la percepción completamente normal e inalterada de los sentidos". (36). Por eso habla de delirio de significación. Según el "material sensorial" con que se experimenta esta significación, se habla de percepciones delirantes, representaciones delirantes, recuerdos delirantes, cogniciones delirantes, etc.

Es, pues, el hecho de ser comprensibles o no lo que diferencia las ideas deliroides, las ideas sobrevaloradas y las creencias normales de las ideas 
delirantes genuinas. Mientras que éstas son inmediatas, incomprensibles e implican un cambio en la personalidad o "conjunto individualmente distinto y característico de las relaciones comprensibles de la vida psíquica." (37), las primeras son comprensibles, pues surgen de los afectos, las pseudopercepciones y otras experiencias, y de la personalidad, resultan del juicio o de la reflexión y no alteran la personalidad.

Para muchos autores, y es la manera en que suele transcribirse en los textos al uso, la concepción jaspersiana del delirio parte de lo siguiente: "Ideas delirantes se llama incluso de modo vago a todos los juicios falseados que tienen los siguientes caracteres externos en una cierta medida -no circunscripta (sic) con precisión: 1. La convicción extraordinaria con que se afirma uno en ellas; la certeza subjetiva comparable. 2. La condición de no influible por la experiencia y por las conclusiones irrefutables. 3. La imposibilidad del contenido." (38). Pero es obvio que en este párrafo Jaspers no analiza las características esenciales del delirio ya vistas, sino las características externas, propias del juicio con que es expresado. Así, por ejemplo, la convicción e incorregibilidad no son características exclusivas del delirio primario, ya que pueden darse en otros fenómenos: las creencias normales pueden ser incorregibles en virtud de la cohesión social que proporcionan; también podrían serlo las ideas sobrevaloradas por la carga afectiva que las sustentan (39).

El principal problema que plantea el concepto jaspersiano de comprensión como criterio clasificador es su escasa objetividad. Bleuler señala al respecto: "Sin embargo, la ausencia de motivos y la 'ultimidad' reside en la propia vivencia del enfermo; el observador halla motivos para ellos en las angustias, temores y esperanzas del paciente. Lo que ocurre es que el comprender o intuir depende más bien de la capacidad del observador que del propio enfermo." (40). En un sentido similar se pronuncia Castilla del Pino: "no haber distinguido Jaspers entre lo comprensible y lo comprendido ha sido el origen de su limitación (...) 'ser comprensible' es una cualidad de la vivencia, mientras que 'ser comprendida' es una categoría que afecta al sujeto que la interpreta, y que forzosamente es otro en la pura investigación fenomenológica". (41) Por otro lado, para este autor, la categoría de primariedad debería radicar en la propia estructura del fenómeno morboso; lo ideal sería poder decir "es primario y, por eso mismo, incomprensible" y no "es primario porque es incomprensible" (42). Asimismo, también considera subjetivo el criterio de compresión, pues "lo que para un determinado investigador resulta incomprensible, para otro no lo es, y es, por tanto, interpretable". (43). Análogamente se pronuncia Berrios: "It is clear, however, that assessment of comprehensibility depends on the state of progress of psychological theory: delusions incomprehensible today may not be so tomorrow." (44). Por otro lado, lo que Jaspers denomina "continuidad de sentido" se refiere solamente a la vida psíquica consciente, pues, según él, lo inconsciente no es vivenciado o experimentado por el sujeto y, por tanto, no es susceptible de verificación (45). 
Se eluden de este modo los motivos inconscientes de la conducta, por lo que "sin el supuesto de una dimensión inconsciente de la mente, la actividad psicológica se disuelve en un despliegue absurdo de hechos desconectados, fortuitos e incluso extravagantes". (46)

¿Cuál es la posición de Jaspers respecto a la naturaleza del delirio paranoico? La naturaleza del delirio, siguiendo los criterios antes vistos, dependerá de la compresibilidad de su contenido. En su trabajo Delirio celotípico, contribución al problema: ¿'desarrollo' de una personalidad o 'proceso'?, de 1910 (47) realiza algunas consideraciones al respecto. En este trabajo, Jaspers, para quien la descripción del caso individual es inexcusable -"No nos podemos entender en psiquiatría si la descripción de casos aislados" (48)expone exhaustivamente varios casos de delirio de celos. De ellos, los dos primeros serían procesos psíquicos -aunque no descarta que en su base exista un proceso cerebral-, y los dos siguientes serían desarrollos, ya que las ideas delirantes que los constituyen son comprensibles a partir de la personalidad de los pacientes (49).

\section{La paranoia en Kraepelin}

Hasta la delimitación que hiciera Kraepelin de la paranoia en la edición de 1899 de su tratado, la extensión de este concepto era enorme. Según Séglas, se trataba del concepto psiquiátrico que poseía "la significación más vasta y la peor definida". (50). Es importante para el tema que nos ocupa tener en cuenta la evolución de las ideas de Kraepelin respecto a la paranoia en las sucesivas ediciones de su tratado (51) (52) (53) (54). En la $1^{\text {a }}$ edición, de 1883, habla de "delirio sistematizado (Verrücktheit) primitivo". En la 2a , de 1887, incluye en esta noción: a) las formas depresivas: delirio de persecución alucinatorio, delirio de persecución combinatorio, delirio hipocondríaco y delirio de querulancia; b) las formas expansivas: delirio alucinatorio, combinatorio y Verrutcktheit originaria. En la $4^{a}$ edición, de 1893, adopta el término "paranoia" y agrupa bajo él los "procesos psíquicos degenerativos", incluyendo en ellos la dementia praecox, la catatonía y la dementia paranoïdes. En la $5^{\text {a }}$ edición, de 1896 propone una clasificación que constituyó la base de las que la siguieron; así, entre las "enfermedades mentales constitucionales" está la paranoia, con las formas combinatorias o interpretativas (incluyendo en éstas al delirio querulante) y las formas fantásticas o alucinatorias. La $6^{\mathrm{a}}$ edición, de 1899 es la edición clásica de su tratado, la que se impone en todo el mundo; en ella es donde se diferencian netamente la dementia praecox, la paranoia y la locura maníaco-depresiva. "La paranoia se caracteriza por el desarrollo insidioso, a partir de causas internas, de un sistema delirante durable e inquebrantable, que coexiste con el total mantenimiento de la claridad y el orden del pensamiento, la voluntad y la acción." (55). Solo incluye las formas combinatorias de la clasificación precedente y se trataría de una enfermedad endógena y crónica. La $7^{\text {a }}$ edición, de 1904, no cambia en lo sustancial su concepción de la paranoia. Es en la $8^{\mathrm{a}}$ y última, que aparece entre 1909 y 1913, donde se reestructura en muchos puntos su sistemática Por lo pronto, continúa considerándola un cuadro crónico, de aquí que, aunque 
HISTORIA

reconozca su existencia, quedan eliminadas como no paranoicas todas las formas clínicas consideradas "curables", "abortivas", etc.

Para Kraepelin, la paranoia es un cuadro incurable en el que la temática de las ideas delirantes gira alrededor de dos polos principales: el perjuicio (persecución, celos, hipocondría) y la grandeza (invención, filiación, mística, erotomanía). No existen alucinaciones, pero sí intuiciones delirantes, interpretaciones, falseamiento de los recuerdos e ideas de referencia. A pesar de que su evolución es, como hemos dicho, crónica, no existe nunca, aparte del delirio, otras alteraciones del funcionamiento mental ni, particularmente, deterioro como el que caracteriza a la demencia precoz. Junto a la paranoia, pero netamente diferenciado de ella, Kraepelin describe el delirio querulante; aunque en determinados aspectos se parecen, se diferencian en que: 1) las ideas delirantes de reivindicación están basadas en un perjuicio real, por lo que Kraepelin las consideró psicógenas y no de "causa interna"; 2) la temática delirante está estrechamente relacionada con el perjuicio sufrido, y 3) al contrario que en la paranoia, el pronóstico es frecuentemente favorable.

Desde su formulación inicial, la concepción de Kraepelin sobre la paranoia avanzó hacia la psicogénesis; así, el delirio de grandeza es "la trama suscitada desde la adolescencia hacia proyectos de alto vuelo y proseguidos, de adulto, tras la frustración de aquellos" (56). El carácter tiene solo un papel predisponente, al igual que los acontecimientos vitales. Kraepelin muestra así cierta ambigüedad entre la idea de desarrollo por "causas internas" y la reacción a "causas externas". Por eso, con el tiempo, tendió a borrar las diferencias entre paranoia y delirio querulante: "The differences in the history of the origin of querulant delusion and paranoia, therefore, run out only in the direction of a certain displacement of the relations between external, psychogenic influences and internal morbid causes." (57).

\section{Kretschmer: el delirio de referencia de los sensitivos}

El cometido de DS es para Kretschmer, “... enjuiciar de un modo amplio y lo más completo posible el papel de la base caracterológica y de las acciones vivenciales, y estudiar y describir las intimas relaciones que existen entre una forma caracterológica especial, exactamente delimitada, y una clase especial de formación y elaboración de vivencias, describiéndose sus leyes psicológicas internas." (58).

Como es natural, el libro de Kretschmer no surgió aisladamente, fuera de todo contexto; con anterioridad a la publicación de DS existían numerosos trabajos en los que se trataba de relacionar el delirio con determinados modos de ser. Mas el trabajo de Kretschmer es pionero en un doble sentido; en primer lugar, porque trata de establecer una sistemática caracterológica útil para comprender la génesis de numerosos cuadros psicorreactivos (delirios, neurosis obsesivas, depresión, etc.); en segundo lugar porque, en la aplicación de esta sistemática caracterológica, muestra muy finamente las estrechas relaciones entre un tipo peculiar 
de delirio de referencia y un modo de ser, al que denomina sensitivo. En esto, se puede considerar completamente original.

Aunque -no puede ser de otro modo- los cimientos de la caracterología de Kretschmer asientan en elementos de naturaleza biológica (neurofisiológica) -estímulo-respuesta, conducción de la "energía nerviosa", etc.-, sus derivaciones tienen una clara orientación antropológica, pues, entre otros factores, en su génesis intervienen fundamentalmente las relaciones que el sujeto establece con su medio social; de aquí surgen conceptos tales como los de autoconciencia, autoafirmación, capacidad ética, etc. La lucha del sujeto por el mantenimiento de su autoestima es la clave que permite la comprensión del delirio paranoico. En esto, según W. Kretschmer, E. Kretschmer se aproxima a la psicología del Adler (59). En esta visión antropológica, el concepto de vivencia ocupa un lugar central; Kretschmer lo define como el conjunto de sensaciones y representaciones que producen un afecto. Esto no ha de ser entendido mecánicamente, ya que es el carácter lo que determina el modo de vivenciar la realidad, es decir, si una determinada experiencia afecta al sujeto y de qué modo lo hace. Así pues, debe entenderse la vivencia como la elaboración individual de los acontecimientos vitales; en este sentido, si los constituyentes básicos del carácter están determinados por la predisposición constitucional, para Kretschmer más determinante aún es la relación del sujeto con su entorno social.

Por ello, considera que las "cualidades" del carácter se aprehenden cientí- ficamente "al perseguir, desde el principio hasta el fin el paso de la vivencia por el alma" (60). El camino que sigue la vivencia (estímulo) está predeterminado por las "reacciones del temperamento", esto es, la "capacidad de impresión", la "capacidad de retención", la "actividad intrapsíquica" y la "capacidad de conducción"; por ejemplo, una vez que la vivencia está en el campo de la conciencia, su ulterior destino depende de la capacidad de retención, es decir, de la conservación intrapsíquica de los afectos unidos a representaciones -lo que el psicoanálisis, particularmente el jungiano denominó complejo-. La capacidad de conducción sería aquella capacidad del sistema nervioso de permitir el "flujo de vivencias", y "...la capacidad total del rendimiento del carácter, (es) una magnitud puramente dinámica que se mide por la cantidad total de energía psíquica existente y disponible en cada individuo". (61). Esta cantidad se denomina "fuerza psíquica" y según su grado se distinguen caracteres esténicos o asténicos, así como sus reacciones correspondientes, esténicas y asténicas. Esténico se refiere al sentimiento de superioridad, de energía, de acción, con tendencia a la sobreestimación de sí mismo y agresividad; por el contrario, asténico hace referencia al sentimiento de inferioridad, de debilidad, de padecimiento, tendencia a la subestimación de sí, inseguridad y blandura. Estas son las "capacidades del carácter" - es decir, el temperamento-, y los "hábitos del carácter" descansarían sobre ellas, constituyendo la estructura del carácter. Por ejemplo, las actitudes o "cualidades" éticas serían para Kretschmer aquellos hábitos del carácter referidos a la voluntad. 
Existen cualidades del carácter de naturaleza reactiva $\mathrm{y}$, por lo tanto, adquiridas, y otras de naturaleza puramente biológica. Pero, asimismo, existen "formas reactivas" específicas de determinado carácter, como es el caso del delirio sensitivo de referencia, así como, también, formas inespecíficas. Las primeras, denominadas "formas reactivas psicopáticas" son: las reacciones primitivas, las desviaciones, las reacciones y los desarrollos expansivos, las reacciones y los desarrollos sensitivos y las reacciones puramente asténicas. Estas reacciones corresponden a cuatro caracteres diferentes: primitivo, expansivo, sensitivo y asténico. "...la reacción sensitiva viene especialmente condicionada por el defecto de conducción. La energía psíquica movilizada por la vivencia se 'retiene', se acumula alrededor del grupo emotivo, el cual se convierte en seguida en un cuerpo extraño torturante, sentido conscientemente en el interior de la conciencia". (62). Es de este modo como se da lugar a las representaciones obsesivas de las neurosis obsesivas y al delirio de relación de los sensitivos.

Los factores que determinan la aparición del delirio son el carácter, la vivencia y el medio social; se añaden, asimismo, influencias de naturaleza biológica. Si bien Kretschmer admite una cierta base biológica, una cierta predisposición congénita en los sujetos sensitivos a sufrir síntomas de "agotamiento físico y nervioso" que colorearían el cuadro, así como también la "debilidad de las necesidades sexuales" como circunstancia favorecedora, el conjunto de sus concepciones sobre el delirio de referencia es totalmente de índole psicogenética.

\subsection{El carácter.}

La descripción del carácter sensitivo evoca la psicastenia de Janet. En 1903 Janet (63) describió un tipo de neurosis caracterizada por obsesiones, escrupulosidad, timidez, vergüenza, dudas, sentimientos de incompletud, despersonalización y depresión que, como a renglón seguido veremos, es semejante a la descripción que posteriormente hizo Kretschmer del carácter sensitivo. Dice el autor: "'sensitivo' o 'sensible' corresponde a ciertas naturalezas delicadas que no solo son muy profundamente receptivas $y$ sensibles a sus vivencias, sino que las siguen elaborando interiormente, en silencio, sin que se trasluzca nada al exterior. Este tipo caracterológico posee más componentes asténicos que esténicos" (64). Kretschmer describe como sigue el carácter sensitivo: "Los representantes completamente desarrollados de este grupo son personalidades blandas, de fina percepción, espiritualmente diferenciadas e introvertidas, de sentimientos éticos profundos, con tensiones emotivas ocultas y muy duraderas; son gentes que no asimilan los acontecimientos fuertes ni son capaces de expresarlos libremente, y que poseyendo un gran valor interno, muestran un continente exterior algo inseguro y falto de libertad. La variante de este tipo humano con tendencia a la representación obsesiva queda perfectamente caracterizada por su carácter pedante y muy escrupuloso. La otra variante caracterológica, cuya expresión es el delirio de referencia (...)." (65). Más adelante, en otro pasaje refiere: "Por un lado, muestra una extraordinaria blandura afectiva, debilidad y vulnerabilidad, y, por el otro, cierta ambición y obstinación. 
Los representantes de este grupo caracterológico son, cuando el desarrollo es completo, personas complicadas, muy inteligentes y de gran valía, finas y muy sensibles, de ética escrupulosa, y cuya vida afectiva es íntima, hipersensible; se hallan privados de defensa ante las dureza de la vida, encierran profundamente en su interior sus duraderas y tensas emociones. $\mathrm{Su}$ autoobservación y su autocrítica son finísimas. Estas personas son muy susceptibles y tercas, pero tienen una gran confianza y son muy amorosas; se valoran en mucho, son tímidas, y su presentación personal carece de aplomo. Son introvertidos, pero también asequibles y amables, humildes pero muy ambiciosos y con una gran capacidad social (...) El estado de ánimo de estas personas tan serias tiende a enturbiamientos reactivos de larga duración, aunque en modo alguno es constitucional depresivo, (...).”(66). El rasgo esténico de la personalidad sensitiva permite la proyección de la vivencia culpable y, con ello, la aparición de las ideas delirantes de autorreferencia.

\subsection{La vivencia.}

Sobre el tipo de carácter que acabamos de describir actúa una experiencia vivida por el sujeto de manera humillante y que sitúa ante sus ojos su propia insuficiencia. Estas experiencias traumáticas suelen ser conflictos derivados de actitudes afectivo-eróticas no asumidas por el sujeto y que, por ello, en virtud de su estricta conciencia moral, le suscitan intensos sentimientos de culpa. Ejemplos de ello son la masturbación inveterada, los amores tardíos de solteronas de estrecha moral sexual, determinadas tendencias sexuales, etc. También en su casuística incluye Kretschmer casos de fracaso profesional. "La vivencia de la insuficiencia vergonzante, de la inferioridad moral, actúa patogénicamente sobre el carácter sensitivo." (67). En cualquier caso, estas experiencias conflictivas han de ser interpretadas en función del carácter, es decir, desde el sujeto: “(...) la vivencia que hemos llamado, por ejemplo, 'amor desgraciado', resulta fundamentalmente distinta según una forma vivencial subjetiva para las diversas personalidades psicopáticas." (68); de aquí que, para la personalidad sensitiva, un amor desgraciado constituya una "derrota vergonzosa" . "Es la vivencia interior lo que hace al paranoico (...) La vivencia indignante hace al paranoico combativo; la vivencia retenida, al paranoico sensitivo, y la vivencia fantástica, al paranoico desiderativo. Así hemos resuelto la clave del problema de la paranoia, al afirmar que hay paranoicos, no paranoia." (69).

\subsection{El ambiente.}

A los factores estudiados se añaden las influencias ambientales, las cuales, para Kretschmer, no son imprescindibles sino solo coadyuvantes en la patogenia del delirio: aislamiento, poblaciones pequeñas de moral convencional y fuerte control social, etc. "La fórmula según la cual el ambiente favorece la génesis de la afección es la siguiente: tensión de la propia estimación en una circunstancia humillante." (70).

\subsection{Formas sindrómicas.}

El carácter sensitivo da lugar a cuatro formas sindrómicas: la paranoia sistematizada, el delirio sensitivo agudo, la neurosis de referencia y las formaciones 
HISTORIA

delirantes inconstantes del tipo de la neurosis obsesiva. La forma sistematizada de la paranoia es la más frecuente. El cuadro clínico consiste en un delirio sistematizado en el cual es típica la oscilación de la certeza sobre las ideas delirantes, que va desde la convicción absoluta a las meras ideas sobrevaloradas (que, por cierto, en muchas ocasiones Kretschmer califica erróneamente de "obsesivas"). El delirio aparece por ello muy influible por la crítica. Entre los casos que expone Kretschmer, los de $\mathrm{H}$. Reiner y el Dr. Kluge son paradigmáticos. En el delirio sensitivo agudo, sobre el delirio sistematizado aparecen bruscamente episodios con temas de influencia, de transmisión del pensamiento, sentimientos de extrañeza, etc. El caso Hélène sería un ejemplo típico. La neurosis de referencia consiste en un cuadro constituido a expensas, más que de ideas delirantes, de ideas sobrevaloradas, muchas veces con un tinte hipocondríaco. Se corresponde con los casos de delirio de los masturbadores. Por último, la formación delirante inconstante es rara. Se trata de un estado de nerviosismo intenso sobre el que surgen, de vez en cuando, ocurrencias delirantes no sistematizadas de muy breve duración. Un caso típico es el del tornero de Friedman.

5. Antecedentes de El delirio sensitivo de referencia

¿Qué supone DS frente a la concepción kraepeliniana de la paranoia? En primer lugar, el reconocimiento de casos curables; en segundo lugar está la consideración reactiva, psicógena de este trastorno. Como se ha visto anteriormente, una de las cuatro formas clínicas, la más frecuente, que adopta el delirio sensitivo es el de la paranoia: "En esto casos se encuentra un delirio de referencia sistematizado, conservándose la lógica formal y la reflexión." (71). En lo que respecta a la concepción kraepeliniana de la paranoia ( $c f r$. cap. 3), para Kretschmer ambos cuadros guardan numerosas relaciones "psíquicas", esto es, en sus rasgos caracteriales, conformándose entre estos "una trama finísima e insoluble". De aquí que el conjunto de todas las formaciones delirantes reactivas, y la paranoia de Kraepelin sería un tipo de éstas, deberían recogerse, según Kretschmer, en un solo "grupo" patológico. (72).

En los dos sentidos antes señalados, como casos curables y reactivos, son varios los antecedentes del libro de Kretschmer (73). Por ejemplo, la autopsicosis inducida basada en una idea sobrevalorada, de Wernicke; la paranoia periódica, de Gierlich; las figuraciones delirantes de los degenerados, de Birnbaum, y la formación delirante catatimica, de Maier. Todos ellos son cuadros que, para Kretschmer permiten situar la paranoia dentro de los desarrollos de la personalidad, esto es, como casos comprensibles. Asimismo, en Francia, en 1898, Janet observó delirios de persecución, a los que denominó paranoia rudimentaria, en sujetos que presentaban los síntomas de lo que llamó obsesión de los escrupulosos. Este cuadro aparece como reacción a acontecimientos traumáticos y su predisposición constitucional es la misma que la de los psicasténicos. 


\subsection{Gaupp y el caso Wagner.}

Mas, fundamentalmente, en relación con el tema que estamos tratando, hay que tener en cuenta, por un lado a Friedman y, por otro, a Gaupp. En cuanto al primero, del que Kretschmer transcribe en su libro un caso como ejemplo de "deliro erótico de referencia de las solteronas" (74), en sus Contribuciones a la teoría de la paranoia, describe casos de paranoias leves como subgrupo de la paranoia verdadera de Kraepelin. Se trata de delirios que surgen sobre una predisposición caracterial ("sensibles, orgullosos, exaltados"), pero, sobre todo, es la reacción a un acontecimiento lo que da lugar al delirio. El problema de Friedman es que, al faltarle una suficiente teoría del carácter, sus cuadros clínicos hacen excesivo hincapié en dicho acontecimiento.

En este sentido, esto es, en el desarrollo de una base caracterológica que permite comprender la génesis del delirio, avanzó mucho más Gaupp; precisamente, son sus descripciones las que más influencia ejercieron en Kretschmer. Por eso merece la pena detenernos brevemente en este autor. En la concepción de Gaupp acerca de la paranoia destaca: 1) el reconocimiento de las formas agudas; 2) la posibilidad de que sea provocada por conflictos del sujeto con su entorno, y 3) el importante papel de la disposición caracterial.

Gaupp describe un carácter, al que denomina disposición depresivoparanoica, que se asemeja a la psicastenia de Janet y al carácter obsesivo. Se trata de sujetos de 25 a 45 años, de buen talante, temerosos, inseguros, escrupulosos y muchas veces pedantes, pero a la vez reflexivos, autocríticos y exentos de combatividad. Sobre este carácter surge un delirio de autorreferencia de evolución insidiosa, con certidumbre oscilante y con mayor o menor relación con una circunstancia exterior, pero ésta, al contrario de los casos descritos por Friedman, no ocupa un lugar preeminente. Para Gaupp, la base caracterial es tan importante que llama a este cuadro formación delirante caracterógena. "Los rasgos generales de la paranoia abortiva, según Gaupp, son: disposición básica psicasténico-neuróticoobsesiva; durante la enfermedad, formación delirante insidiosa, afecto pasajeramente depresivo-temeroso, reconocimiento parcial de la enfermedad, oscilaciones del curso y ausencia de desolación egoísta”. (75). La expresión "abortiva" con que Gaupp califica estos cuadros se refiere a la fluctuación de los síntomas y la escasa fijeza de las ideas delirantes. Mas, según Kretschmer, a pesar de la importancia del carácter de base, como para Gaupp la vivencia no influye de un modo decisivo en el cuadro patológico, sus casos se tratarían más bien de procesos, más que de desarrollos: "Nuestros casos sensitivos constituyen reacciones y desarrollos, $\mathrm{y}$, de un modo idéntico, los casos de Gaupp representan procesos..." (76).

Aparte lo anterior, en el caso princeps descrito por Gaupp en 1914, el del maestro Wagner (77), el delirio surge también a partir de una determinada estructura de la personalidad, pero ésta no es como la anteriormente descrita; de hecho, en DS Kretschmer sitúa el caso Wagner como una variante caracterológica 
HISTORIA

de delirio sensitivo (78) en el que el rasgo esténico del carácter se consolida tras un periodo de larga evolución de un delirio típicamente sensitivo, dando paso a un delirio expansivo. Para Kretschmer, Wagner es la imagen típica de la paranoia de Kraepelin, pero una paranoia de naturaleza psicógena, aunque no hay que desdeñar el papel que en este caso Gaupp atribuye a la herencia.

Bajo la presión de las ideas delirantes, Wagner asesinó a su familia (esposa y cuatro hijos) y a nueve personas más del pueblo donde había vivido. En el extenso estudio que como perito Gaupp realizó sobre el paciente, se exponen los principales rasgos de carácter que sus amigos y conocidos le atribuyen: "pagado de sí mismo", "presunción y arrogancia"; "vanidoso y autosuficiente"; "altivo, afectado y antinatural", autosuficiente y pretencioso"; "exagerado, soñador y muy frío"; "megalomanía”, etc. (79). Asimismo, en su "Informe general y psicocriminológico" (80), basado en las entrevistas mantenidas con Wagner, así como en los tres volúmenes de la Autobiografía de éste y otros escritos suyos (fundamentalmente cartas y varias obras dramáticas), Gaupp destaca los siguientes rasgos de la personalidad del enfermo: "La predisposición patológica se manifiesta mucho antes de la irrupción del delirio a través de una serie de síntomas. Entre estos síntomas se cuentan, en el caso Wagner: una hipersensibilidad innata, una gran irritabilidad afectiva, una autosuficiencia desmesurada, una tendencia general a sentirse postergado e incluso perseguido, cierta discordancia de las funciones psíquicas, una manera de pensar y de sentir completamente egocéntrica cuya divisa es: 'Tua res agitur', los múltiples síntomas de la neurastenia, una tonalidad afectiva hipocondríaca cuando aparece algún trastorno físico, un impulso sexual anormalmente intenso..." (81). Y es a partir de esta personalidad que, según Gaupp, se desarrolla el delirio: “...este delirio es, a su vez, el producto de una transformación psíquica profunda $\mathrm{y}$ generalizada: no es un añadido secundario en la personalidad de Wagner, sino su núcleo". (82).

El origen remoto del delirio radica en los sentimientos de culpa a que dieron lugar las prácticas onanistas que Wagner comenzó a los 18 años de edad, intensificados por las conductas zoofílicas comenzadas 9 años más tarde. Dice Gaupp a este respecto: “...y esas violentas conmociones afectivas -la culpa por la zoofilia- relanzaron el proceso patológico que había perturbado largo tiempo su espíritu: la referencialidad personal enfermiza, el delirio interpretativo". (83).

La relación dinámica entre personalidad y delirio en Wagner y, fundamentalmente, cómo el delirio constituye la negación y proyección de sentimientos de culpa, son agudamente vistos por Gaupp: "Wagner comenzó a proyectar hacia fuera la angustia y los remordimientos que le atormentaban. Dicho en términos más simples: la mala conciencia produce en las personas cavilosasy críticas el sentimiento de que así como se consideran a sí mismas seres distintos y de algún modo indignos, los demás también han de notar en ellas esa diferencia y suponer cosas extrañas. Y entonces surge el recelo". (84). Es decir: 
HISTORIA

“...la percepción del mundo exterior es falseada bajo los efectos de un sentimiento exacerbado de angustia y culpabilidad". (85). Como hemos dicho más atrás, el móvil de los asesinatos cometidos por Wagner radica en su delirio de referencia: "Pero resulta que él, un hombre altivo y orgulloso que miraba a la multitud desde lo alto de su arrogancia intelectual, solo era objeto de mofas y sarcasmos, una especie de 'bufón' destinado a divertir a la gente. Y la cólera vino así a sumarse al pesar y la aflicción, ...” (86).

Para Gaupp es indudable que el delirio de Wagner constituye un caso claro de paranoia: "El síndrome en cuestión es el delirio de persecución crónico y sistemático: la paranoia." (87); y, al igual que Bleuler (vid. más adelante), considera que la certeza delirante deriva de una patología de la afectividad: "Esto nos permite comprobar asimismo que toda convicción surgida de una afectividad patológica es inaccesible a cualquier refutación racional”. (88).

\subsection{Bleuler y la teoría de la afectividad.}

Para Bleuler (89), pionero en la concepción psicodinámica de la psiquiatría clínica, concepción que tomó de Freud a través de Jung durante la estancia de éste en el Burghölzli, el estudio de la paranoia debe ser enmarcado en el de la afectividad en general. De Jung procede la noción de complejo de tonalidad emocional como conjunto de representaciones, recuerdos, ideas, etc., centrados sobre una experiencia afectiva. Jung aplicó esta teoría a la interpretación de los síntomas esquizofrénicos (90) y Bleuler la aplicó primero a la génesis del delirio paranoico y más tarde también a la esquizofrenia (91). Hay que decir que ya en 1885 Charcot había hablado de la existencia de "ideas fijas" inconscientes, concepto que Janet (idée fixe subconsciente), Freud ("reminiscencias traumáticas") desarrollarían más tarde (92).

Según Bleuler, la afectividad se sustenta en dos tipos de reacción: a) general del humor u holotímica, como, por ejemplo, ocurre en la manía y en la melancolía, y b) catatímica, ligada a acontecimientos vitales, creándose complejos representativos formados en torno a esos acontecimientos (93). Bleuler también explica el delirio como una reacción del sujeto ante conflictos vitales, pero no parte de una predisposición constitucional, sino que, para él, como mucho, el temperamento prestaría colorido al cuadro. De este modo, la paranoia podría darse en gran variedad de disposiciones constitucionales, pues lo importante aquí no es tanto la personalidad como el conflicto interno que da lugar al delirio; determinados conflictos sobrepasan la capacidad del sujeto, humillándolo éticamente; frente a ello, el sujeto reacciona negándose a aceptar su incapacidad (delirio de grandeza) o atribuyendo el motivo de su fracaso a los demás (delirio de persecución). El delirio entraña una distorsión catatímica de la realidad conflictiva, pues en la paranoia no existe una perturbación general u holotímica del humor; los estados afectivos que vemos en su curso son secundarios al contenido de las ideas delirantes.

El error de juicio que implica el delirio no difiere mucho del que comete el sujeto sano cuando es presa de una pasión, 
solo que en el paranoico la afectividad ligada a los complejos representativos es especialmente estable, lo que hace que el error no sea corregido y se extienda por propagación. "Esta superación de la lógica por los afectos presupone que, en el paranoico, la afectividad tiene que poseer una fuerza conmutadora demasiado intensa en relación con la firmeza de las asociaciones lógicas." (94). La permanencia del delirio se debe a la permanencia del conflicto existente entre las aspiraciones del sujeto y la realidad. "Lo patológico es solo la fijación del error, en virtud de la cual se transforma éste en delusión, y luego la propagación de la delusión, en virtud de la cual la anormalidad se transforma en paranoia." (95).

Al igual que posteriormente hiciera Kretschmer en DS, Bleuler asigna un componente orgánico a la patogenia del delirio: una "insuficiencia de la energía" y cierta "debilidad sexual". Pero, además, en la paranoia existe un componente esquizoide previo, es decir, una "debilidad asociativa", una tendencia a la escisión que, en virtud del conflicto afectivo, daría lugar al delirio: "La esquizofrenia y la paranoia, en suma, aparecen sintomatológicamente como nacidas de una misma raíz (la esquizopatía), a la cual se añade todavía, en la esquizofrenia, un proceso físico, y en la paranoia, sólo la formación delusiva psicógena, consecutiva a la combinación con un determinado carácter." (96)

Siguiendo a Kahn (97), Bleuler piensa que muchos paranoicos han sufrido un proceso esquizofrénico precoz que ha dejado en ellos un defecto muy leve, sobre el cual se establece la paranoia:
"El proceso esquizofrénico produce una debilidad asociativa que permite, incluso a una afectividad poco intensa, adquirir una influencia patológica sobre las asociaciones." (98). De aquí que piense que en la paranoia existe un "componente disposicional" que la introduce en el "círculo de formas esquizoides" (99): “(...) las formas graves de esquizoidia conducen a la esquizofrenia; las formas algo menos graves, si se asocia a ellas la correspondiente constitución afectiva, a la paranoia; las formas todavía más leves caracterizan a los psicópatas esquizoides, $\mathrm{y}$ en los individuos normales, el 'componente esquizoide' de la psique está distribuido en la medida conveniente." (100). Pero en todos los casos, incluso en la esquizofrenia o en la parálisis general, el sistema delirante está condicionado psicógenamente, es decir, nace por mecanismos psíquicos y en virtud de necesidades psíquicas.

\subsection{Sérieux y Capgras: la constitución} paranoica y la afectividad.

En la psiquiatría francesa de principios del siglo XX destaca el libro de Serieux y Capgras Les folies raisonantes. Le délire d'interpretation, de 1909 (101). En este trabajo sus autores aceptan la definición de paranoia propuesta por Kraepelin, considerando sinónimos delirio de interpretación y paranoia. Sin embargo, en la medida en que el delirio asienta sobre una determinada personalidad, a la que denominan constitución paranoica, pasa a ser considerado de naturaleza psicógena. Esta constitución paranoica es fundamental para la intelección de la génesis del delirio, el cual no sería más que la exageración de ciertas tendencias 
preexistentes en el sujeto; es decir, usando la terminología de Jaspers, el delirio sería un desarrollo de dicha constitución: "Point de rupture entre la personnalité antérieure du sujet et la personnalité de l'interprétateur. Celle-ci n'est que l'épanouissement de la première qui, persistant avec ses tendences, son caractère, ses modes de réaction accoutumés, influence l'elaboration du délire, le choix des conceptions, et l'activité tout entiere." (102)

La constitución paranoica comprende anomalías intelectuales y afectivas; las primeras consisten en una disminución de la autocrítica, "une paralogique circonscrite qui contraste avec la conservation de la lógique formell". (103); las segundas tienen su núcleo en la hipertrofia del yo. En el estadio prepsicótico, el paranoico es un sujeto inadaptado socialmente, debido en parte a su tendencia a falsear todos aquellos hechos de la realidad contrarios a la alta opinión que tiene de él mismo. Esta inadaptación social deriva también de su hiperesthésie psychique, que, para los autores, es lo más congénito de la constitución paranoica: se trata de sujetos emotivos, suspicaces, recelosos y soñadores. Estas actitudes se mantienen contenidas hasta que bien un choque emocional intenso, bien repetidas heridas al amor propio hacen emerger lo que denominan idée prévalent, la cual orientará y dará coherencia al conjunto de interpretaciones falsas que constituye la psicosis. En cualquier caso, la causa provocadora pone en conflicto con el medio a un sujeto previamente mal adaptado en virtud de su personalidad.

Por lo demás, los autores siguen a Bleuler, ya que, como este autor, hablan de afectos ligados a representaciones, a lo que llaman complexus idéo-affectif prédominant, complejo con el que el paciente pone en relación hechos de la vida cotidiana, resultando así el delirio de referencia. La hipertrofia del yo, otro rasgo fundamental de la personalidad del delirante, es, para los autores, una consecuencia del complejo ideo-afectivo. En definitiva, "Le délire d'interprétation est, en resume, une psychose constitutionelle, qui se développe grâce à une anomalie de la personnalitéé caracterisée par l'hypertrofie ou l'hyperesthésie du moi et par la défaillance circonscrite de l'autocritique. Sous l'influence des conflits sociaux déterminés par la inadaptabilité au milieu, cette constitution psychique anormale provoque la prédominance d'un complexus idéoaffectif, sa persístanse et son rayonnement." (104)

\subsection{Freud: paranoia y homosexualidad.}

Los trabajos de Freud más importantes sobre la dinámica de la psicosis, Neurosis y psicosis y La pérdida de la realidad en la neurosis y la psicosis son de 1924 (105). Otro trabajo a tener en cuenta es Sobre algunos mecanismos neuróticos en los celos, la paranoia y la homosexualidad, de 1922 (106). Todos, por tanto, posteriores a la aparición de DS. En 1896, en su Nuevas observaciones sobre las neuropsicosis de defensa apunta ya a algunos aspectos básicos de la dinámica del delirio: "Desde hace mucho tiempo vengo sospechando que también la paranoia -o algún grupo de casos pertenecientes a la paranoia- es una neurosis de defensa, surgiendo, como la histeria y las representaciones obsesivas, de la represión de recuerdos penosos, 
HISTORIA

y siendo determinada la forma de sus síntomas por el contenido reprimido." (107). En este trabajo Freud emplea por primera vez el concepto de proyección como mecanismo psicológico fundamental para explicar la génesis del delirio: "En la paranoia, el reproche es reprimido por un procedimiento al que podemos dar el nombre de proyección, transfiriéndose la desconfianza a otras personas." (108).

En 1907 publica El delirio y los sueños en la "Gradiva" de W. Jensen, donde hace ver la similitud existente entre los mecanismos implicados en la fantasía, los sueños y el delirio. El cometido común de estos fenómenos psíquicos es la realización de deseos reprimidos: “(...) se caracteriza -el delirio- por el hecho de que en él adquieren las "fantasías" el supremo dominio; esto es, encuentran fe en el sujeto e influyen en sus actos." (109). Y también. "representémonos ahora a las imágenes oníricas como creaciones delirantes fisiológicas del hombre, (...)" (110). Y, por último, "El sueño y el delirio proceden de la misma fuente; esto es, de lo reprimido, y el sueño es, por decirlo así, el delirio fisiológico del hombre normal." (111)

En 1911 publica Freud su trabajo princeps sobre la dinámica del delirio; se trata de su Observaciones psicoanaliticas sobre un caso de paranoia ('dementia paranoides') autobiograficamente descrito (112), basado en Memorias de un neurótico, publicadas por el magistrado Schreber en 1903. Aunque por muchas razones es dudoso el diagnóstico de paranoia del caso Schreber, la ausencia de defecto en el curso evolutivo así como la tendencia a la sistematización del delirio, en el que domina el tema de redención de la humanidad a partir de la transformación en mujer, lo acercan a la paranoia. El análisis del caso es interesante como modelo de interpretación dinámica del delirio a partir de los diversos mecanismos del funcionamiento mental (desplazamiento, condensación, proyección...) que ya describiera en su Interpretación de los sueños (113). En el caso del delirio, estos mecanismos se aplican, según Freud, como defensa frente a tendencias homosexuales encubiertas que el sujeto es incapaz de reconocer y asumir: “(...) el motivo de la enfermedad fue la aparición de una fantasía optativa femenina (homosexual pasiva) (...). Contra tal fantasía se alzó por parte de la personalidad de Schreber, una intensa resistencia, y la defensa, que quizá hubiera podido adoptar otras formas distintas, escogió, por razones que desconocemos, la del delirio persecutorio. El hombre añorado se convirtió en perseguidor, y el contenido de la fantasía optativa, en el de la persecución." (114). De este modo, el delirio sería un intento del sujeto de restablecer su autoestima, amenazada por la emergencia de impulsos homosexuales. Concretamente, Freud lo interpreta como un intento de Schreber de superar el complejo paterno, especialmente su componente homosexual pasivo; mediante el delirio, se protege de impulsos homosexuales originados en su actitud infantil hacia la figura paterna. (115)

Freud piensa que esta interpretación es generalizable a todos los casos de paranoia: “(...) toda una serie de casos de la paranoia (...) descubriendo, con sorpresa, cuán claramente dejaban 
ver todos ellos, en el punto central del conflicto patológico, la defensa contra el deseo homosexual, y cómo tales sujetos habían fracasado todos en el sojuzgamiento de su homosexualidad inconscientemente intensificada." (116). Aunque estaba dispuesto admitir los límites de dicha interpretación: "Hemos, pues, de estar preparados a limitar eventualmente nuestra afirmación a un único tipo de la misma." (117). Las fórmulas propuestas por Freud para explicar la dinámica del delirio son como sigue:

a) En el delirio de persecución: "Yo (un hombre) le amo (a un hombre)", que se transforma en "No le amo, le odio", que, por medio de una proyección, se transforma en "El me odia (me persigue), lo cual me da derecho a odiarle", es decir, "No le amo; le odio, porque me persigue." (118)

b) En el delirio erotomaníaco: "Yo no le amo a él; amo a ella." Que por medio de una proyección se transforma en "Advierto que ella me ama." Que finalmente queda: "'Yo no le amo a él; la amo a ella, porque ella me ama." (119)

c) En el delirio de celos la fórmula sería: "No soy yo quien ama al hombre; es ella quien le ama." (120)

d) En el de grandeza: "No amo en absoluto, no amo a nadie." Que equivale a "Sólo me amo a mí mismo." (121)

Como hemos dicho, el principal mecanismo psicológico implicado en la paranoia es la proyección: mediante él "es reprimida una percepción interna, y en sustitución suya surge en la conciencia su propio contenido, pero deformado y como percepción externa." (122) Y, asimismo, Freud destaca que la finalidad del delirio es la sobrevaloración del yo: "Deduciremos, pues, que en la paranoia la libido libertada (sic) es acumulada al yo, siendo utilizada para engrandecerlo." (123). En suma, existiría en la paranoia una fijación con ulterior regresión al narcisismo: “(...) y concluimos que el retroceso desde la homosexualidad sublimada hasta el narcisismo revela el alcance de la regresión característica de la paranoia." (124)

\section{Epílogo}

Cuando Kretschmer hace hincapié en el hecho, incontrovertible para él, de que "no existe la paranoia, sino sujetos paranoicos", está planteando ya la necesidad de lo que más tarde llamaría diagnóstico multidimensional, en virtud del cual se abordarían los distintos factores -genéticos, caracteriales, ambientales, etc.- que dan lugar al cuadro clínico. En la actual consideración biopsicosocial del sujeto, reflejada en el diagnóstico multiaxial de los sucesivos DSM, se recoge en gran medida esta idea. Dice Kretschmer en este sentido: "Es poco fructífero clasificar a los paranoicos en cualquier sistema usual. Sólo pueden ser comprendidos como personas totales." (125).

Es muy discutible la existencia de factores orgánicos en la génesis del delirio paranoico; sí parece obvia la existencia de una caracteropatía de base, la cual, ante determinadas situaciones vitales 
conflictivas, haría al sujeto vulnerable al mismo; como dice Castilla del Pino, “...al delirio se "llega" y, por tanto, como error que es, como error del modo de interpretar la realidad y su propia realidad como sujeto, en la biografía del delirante está la clave de por qué ha de adoptar una visión delirada de la realidad y por qué ésta tiene un carácter definitivo e incorregible. El delirio salva al delirante del sufrimiento que el estar en la realidad, y la aceptación de sí mismo a que ella le obliga, le deparan." (126) Parece ser que los sujetos paranoides, los inseguros o evitativos y los esquizoides serían los más vulnerables (127). Cuál sea el contenido del conflicto que se intenta subsanar mediante el delirio creemos que depende de los valores sociales dominantes en cada momento histórico; hoy en día, por ejemplo, es excepcional ver en la consulta un delirio de masturbadores, cosa habitual antaño; asimismo, hasta no hace mucho veíamos con relativa frecuencia delirios de referencia en sujetos con tendencias homosexuales no asumidas.

En el presente trabajo hemos podido comprobar cómo diferentes autores de muy diversa adscripción epistemológica, y reconocidos como clásicos de la psiquiatría, coinciden en la consideración del delirio como una defensa que el sujeto levanta frente al cuestionamiento de su autoestima en situaciones de conflicto. Asimismo, muchos de estos autores coin- ciden en la índole emocional, afectiva, del conflicto. Recientes investigaciones empíricas apuntan también en esta dirección; por ejemplo, Dudley y cols. (128) sugieren que el modo de razonar del sujeto delirante es similar al del sujeto sano cuando éste se siente sobrecargado emocionalmente. Mujica-Parodi y cols. (129) concluyen lo mismo y sugieren que en el sujeto delirante está activado un mecanismo cognitivo que en el sano solo se activa bajo el estrés o el miedo; en este sentido, para estos autores, el delirio podría constituir un intento por parte del sujeto de explicarse sentimientos de miedo o ansiedad que no poseen para él un referente claro. Todo parece indicar, por tanto, que la afectividad influye en la génesis del delirio.

Para terminar añadiré que toda teoría del delirio que pretenda poseer suficiente valor heurístico exige como punto de partida un modelo de sujeto, única manera de dar cuenta de los siguientes dos hechos fundamentales: 1. El sujeto delirante no delira sobre cualquier tema. 2. En la trama que constituye el delirio, el sujeto delirante es siempre protagonista.

\section{Agradecimientos.}

Agradezco a los profesores J. Lázaro, R. Luque y V. Sánchez sus valiosas sugerencias. 
HISTORIA

\section{BIBLIOGRAFÍA}

1. Kretschmer, E. El delirio sensitivo de referencia. Contribucióón al problema de la paranoia y a la teoría psiquiátrica del carácter. Madrid: Triacastela. 2000. (Contiene introducción y capítulo adicional de W. Kretschmer).

2. Gaupp, R. El caso Wagner. Madrid: Asociación Española de Neuropsiquiatría. 1998. 1ª edic. en alemán de 1914.

3. Escande $\mathrm{M}$ et Bonet $\mathrm{B}$, "Le délire de relation sensitif de Kretschmer. Aspects historiques et modernes." Sem. Hôp. Paris. 1985. LXI, 14, 893-899.

4. Cfr. Von Wright, G.H. Explicación y comprensión. Madrid: Alianza. 1980. Contiene un buen núúmero de referencias.

5. Martín Santos, L. Dilthey, Jaspers y la comprensión del enfermo mental. Madrid: Paz Montalvo. 1955. p. 7.

6. Para una revisión de la historia del problema de la etiología en psiquiatría, vid. Berrios, G.E. "La etiología en psiquiatría: aspectos conceptuales e históricos". En Luque Luque, R. y Villagrán Moreno J.M. Psicopatología descriptiva: nuevas tendencias. Madrid: Trotta. 2000. Asimismo, para una crítica de los conceptos endógeno vs. exógeno, reactivo, etc. vid. Lewis, A. "'Endogenous' and 'exogenous': a useful dichotomy?" Psychological Medicine, 1971, 1, 191-196. También, Heron, M.J. "A note on the concept endogenous-exogenous". Brit. J. Med. Psychol. 1965, 38, 241-245.

7. Bercherie, P. Los fundamentos de la clínica. Historia y estructura del saber psiquiátrico. Buenos Aires: Ediciones Manantial. 1986.

8. Alvarez, J.M. "De nuevo la paranoia". En Alvarez, J.M. y Colina, F. Clásicos de la paranoia. Madrid: Dor. 1997.

9. Kretschmer, E. Constitución y carácter. Investigaciones acerca del problema de la constitución y de la doctrina de los temperamentos. Barcelona: Labor. 1961. $1^{\text {a }}$ edic. en alemán de 1921.

10. Lacan. J. De la psicosis paranoica en sus relaciones con la personalidad. 1984. México: Siglo XXI. p. 49. Sub. en el texto.

11. Kretschmer, E. Op. cit. p. 451. Sub. en el texto.

12. Ibid.

13. Idem. p. 452. Sub. en el texto.

14. Jaspers, K. Psicopatología general. 1980. Buenos Aires: Beta. $1^{a}$ edic. en alemán de 1913. p. 726.

15. Lacan, J. Op. cit. p. 41.

16. Idem. p. 29.

17. Para la influencia de la Verstehen en Jaspers, vid. Walker, C. "Delusion: What Did Jaspers Really Say?”. British Journal of Psychiatry. 1991. 159 (suppl. 14), 94-103.

18. Castilla del Pino, C. "Interpretación, interpretado, intérprete". Theoría, 1992, VII, 16-18 1353-1366.

19. Von Wright, G.H. Op. cit. p. 24.

20. Martín Santos, L. Op. cit.

21. Idem. p. 258.

22. Jaspers, K. Op. cit. p. 357.

23. Martín Santos, L. Op. cit.

24. Jaspers, K. "Las relaciones comprensibles de la vida psíquica (psicología comprensiva)". En Jaspers, K. Op. cit. $2^{\mathrm{a}}$ parte.

25. Jaspers, K. Op. cit. p. 447. Sub. en el texto.

26. Idem. 
HISTORIA

27. Jaspers, K. "Relaciones causales y 'comprensibles' entre destino y psicosis en la demencia precoz (esquizofrenia)”. En Escritos psicopatológicos. Madrid: Gredos. 1977. Este artículo, de 1912, es, por tanto, anterior a la Psicopatología general, de 1913, donde es citado en p. 352.

28. Berrios, G.E. The history of mental symptoms. Descriptive psychopathology since the nineteenth century. Cambridge: Cambridge University Press. 1996.

29. Jaspers, K. Psicopatología general. cit.

30. Idem. p. 116.

31. Ibid. Sub. en el texto.

32. Idem. p. 118.

33. Idem. p. 119.

34. Ibid.

35. Idem. p. 123

36. Ibid.

37. Idem. p. 495. Sub. en el texto.

38. Idem. p. 119. Sub. en el texto.

39. Una buena exposición de las concepciones de Jaspers sobre el delirio puede verse en Walker, C. Op. cit.

40. Bleuler, E. Tratado de psiquiatría. Madrid: Espasa-Calpe. 1971. p. 537.

41. Castilla del Pino, C. Un estudio sobre la depresión. Fundamentos de antropología dialéctica. Barcelona: NeXos. 1991 ( $1^{\text {a }}$ edic. de 1966 en Ediciones de Bolsillo). p. 16-17. Sub. en el texto.

42. Castilla del Pino, C. "Para la estructura de la idea delirante primaria". Actas Luso-Españolas de Neurología y Psiquiatría. 1954. XIII, 4 (257-262).

43. Castilla del Pino, C. "Crítica a la razón psicopatológica". En Castilla del Pino, C. y Ruiz-Vargas, J.M. Aspectos cognitivos de la esquizofrenia. Madrid: Trotta. 1991.

44. Berrios GE "Delusions as 'Wrong Beliefs': A conceptual History". British Journal of Psychiatry. 1991. 159 (suppl. 14), 6-13. p. 10.

45. Jaspers, K. Op. cit.

46. Erdelyi, M. H. Psicoanálisis. La psicología cognitiva de Freud. Barcelona: Labor. 1987. p. 64 .

47. Jaspers, K. "Delirio celotípico, contribución al problema: ¿"desarrollo de una personalidad" o “proceso"?". Escritos psicopatológicos. Madrid: Gredos. 1977.

48. Idem. p. 111.

49. Idem. p. 172-173, especialmente nota 13 para la descripción de diferentes tipos de ideas sobrevaloradas.

50. Séglas, "La paranoia, historique et critique." Arch. Neurol. 1887. Cit. en Lacan J. Op. cit. p. 21.

51. Bercherie, P. Op. cit. cap. 12 y 16.

52. Kraepelin, E. Manic-Depressive Insanity and Paranoia. New Hampshire: Ayer Company, Publishers. 1990 (Reimpresión de la edic. de 1921)

53. Kennet, K.S. "Kraepelin and the Diagnostic of Paranoia. Comprehensive Psychiatry, 1988, 29, 1, 4-11.

54. Lewis, A. "Paranoia and paranoid: a historical perspective". Psychological Medicine, 1970, 1, 2-12.

55. Bercherie, P. Op. cit. p. 112. Sub. nuestro.

56. Cit. en Castilla del Pino, C. Introducción a la psiquiatría. 2. Psiquiatría general. Psiquiatría clínica. Madrid: Alianza. 1980. p. 180. 
HISTORIA

57. Kraepelin, E. Op. cit. p. 212.

58. Kretschmer, E. El delirio sensitivo... Op. Cit. p. 56. Sub. en el texto.

59. Kretschmer, W. "Introducción a El delirio sensitivo de referencia", en Kretschmer, E. Op. cit.

60. Kretschmer, E. Op. cit. p. 59.

61. Idem p. 60. Sub. en el texto.

62. Idem p. 75.

63. Janet, P. Les obsessions et la psychastenie. 1919. París: Alcan. Cit. en Weissmann, P. "Morfinomanía y defensa social. Rev. De la Asoc. Esp. De Neuropsiquiatría. 2001. 78 (113-126). Una descripción más pormenorizada en Jaspers, K. Op. Cit. p. 510-511.

64. Kretschmer, E. Op. cit. p. 84. Sub. en el texto.

65. Idem p. 84

66. Idem p. 241-242. Sub. en el texto.

67. Ibid.. Sub. en el texto.

68. Idem p. 107.

69. Idem p. 289. Sub. en el texto.

70. Idem p. 244.

71. Idem p. 247. Sub. en el texto.

72. Más exhaustivamente, Idem, p. 280-292.

73. Lewis, A. Op. cit.

74. Idem cap. 3 .

75. Idem p. 55.

76. Idem p. 272. Sub. en el texto.

77. Gaupp, R. Op. cit.

78. Kretschmer, E. Op. cit. cap. 7.

79. Gaupp. R. Op. cit. p. 53-63.

80. Idem p. 195 y ss.

81. Idem p. 219.

82. Idem p. $220-221$.

83. Idem p. 202.

84. Ibid.

85. Ibid.

86. Idem p. 204.

87. Idem p. 214.

88. Idem p. 208.

89. Bleuler, E. Afectividad, sugestibilidad, paranoia. Madrid: Morata. 1969. $1^{\text {a }}$ edic. en alemán de 1906.

90. Jung, C.G. Psicología de la demencia precoz. Barcelona: Paidós. 1987. $1^{\text {a }}$ edic. en alemán de 1907.

91. Bleuler, E, Demencia precoz. El grupo de las esquizofrenias. Buenos Aires: Hormé. Sin fecha. $1^{\mathrm{a}}$ edic. en alemán de 1911, en el Tratado de psiquiatría de G. Aschaffenburg.

92. Ellenberger, H.F. El descubrimiento del inconsciente. Historia y evolución de la psiquiatría dinámica. Madrid: Gredos. 1976.

93. Cfr. "Afectividad", en Bleuler, E. Afectividad, sugestibilidad, paranoia. Op. cit.

94. Bleuler, E, Op. cit.

95. Idem p. 155. Sub. en texto.

96. Idem

97. Cit. en Bleuler, E. Op. cit. p. 191.

98. Ibid.. 
HISTORIA

99. Idem p. 199.

100.Ibid.

101. Sérieux, P. et Capgras, J. Les folies raisonnantes. Le délire d'interprétation. Marseille: Laffitte Reprints. 1982. (Reimpresión de la edic. de Alcan, París, de 1909).

102.Idem p. 232.

103.Idem p. 234.

104.Idem p. 240.

105.Freud, S. Ob. Comp. T. VII. Madrid: Biblioteca Nueva.

106.Idem.

107.Idem T. I p. 293

108.Idem p. 298. Sub. en el texto.

109.Idem. T. IV. p. 1307.

110.Idem. p. 1316.

111.Idem. p. 1318.

112.Idem T. IV

113.Idem. T. II.

114.Idem, T IV p. 1510.

115.Fenichel. O. Teoría psicoanalítica de las neurosis. Buenos Aires: Paidós. 1966.

116. Freud. T. IV. p. 1516.

117.Idem. p. 1518.

118.Ibid. Sub. en el texto.

119.Ibid. Sub. en el texto.

120.Idem. p. 1519.

121.Ibid.

122.Idem p. 1520.

123.Idem p. 1523.

124.Ibid.

125.Kretschmer, E. El delirio sensitivo...Op. cit. p. 301.

126.Castilla del Pino, C. El delirio, un error necesario. Oviedo: Nobel. 1998. p. 10

127.American Psychiatric Association. DSM IV. Barcelona: Masson. 1995.

128.Dudley REJ, John CH, Young AW, Over DE "The effect of self-referent material on the reasoning of people with delusions". British Journal of Clinical Psychology. 1997, 36, 575-584.

129.Mujica-Parodi LR, Malaspina D, Sackeim HA, "Logical processing, affect and delusional thought in schizoprenia". Harv. Rev. Psychiatry. 2000, 8 (2), 73-83.

*Psiquiatra

Hospital Universitario Reina Sofía - Profesor Asociado de Psiquiatría

Facultad de Medicina - Córdoba

Dirección para correspondencia:

Equipo de Salud Mental - Avda. de América 1-5 - 14001. Córdoba

E-mail: sr1dipaa@uco.es

Fecha de recepción: 11 de julio de 2001. 